\title{
Proposal for chiral bosons search at LHC via their unique new signature
}

\author{
M. V. Chizhov \\ Centre for Space Research and Technologies, Faculty of Physics, \\ University of Sofia, 1164 Sofia, Bulgaria \\ V. A. Bednyakov and J. A. Budagov \\ Dzhelepov Laboratory of Nuclear Problems, \\ Joint Institute for Nuclear Research, 141980, Dubna, Russia
}

\begin{abstract}
The resonance production of new chiral spin-1 bosons and their detection through the Drell-Yan process at the CERN LHC is considered. Quantitative evaluations of various differential crosssections of the chiral bosons production are made within the CalcHEP package. The new neutral chiral bosons can be observed as a Breit-Wigner resonance peak in the invariant dilepton mass distribution, as usual. However, unique new signatures of the chiral bosons exist. First, there is no Jacobian peak in the lepton transverse momentum distribution. Second, the lepton angular distribution in the Collins-Soper frame for the high on-peak invariant masses of the lepton pairs has a peculiar "swallowtail" shape.

PACS numbers: 12.60.-i, 13.85.-t, 14.80.-j
\end{abstract}

\section{INTRODUCTION}

The gauge interactions are the only well-established fundamental interactions in Nature. Nevertheless, the additional Yukawa interactions of the Higgs bosons are also necessary for a self-consistent construction of the Standard Model (SM). They are still waiting for their experimental verification as a priority part of the CERN LHC program. Besides this, many other programs and searches have been approved [1, 2] to explore further the LHC potential. One of them consists in searching of hypothetical excited fermions $f^{*}$ and their magnetic moment (Pauli) type couplings to ordinary matter

$$
\mathcal{L}_{\text {excited }}^{f^{*}}=\frac{g}{2 \Lambda} \bar{f}^{*} \sigma^{\mu \nu} f\left(\partial_{\mu} Z_{\nu}-\partial_{\nu} Z_{\mu}\right)+\text { h.c. },
$$

where the parameter $\Lambda$ is connected to the compositeness mass scale of the new physics. Up to now their searches have been fulfilled at the powerful colliders, such as LEP [3], HERA [4] and Tevatron [5]. Due to their anomalous type of couplings they lead to a unique experimental signature for their detection.

In this paper we would like to interpret the interactions (11) from a different point of view, introducing the excited boson states

$$
\mathcal{L}_{\text {excited }}^{Z^{*}}=\frac{g}{2 \Lambda} \bar{f} \sigma^{\mu \nu} f\left(\partial_{\mu} Z_{\nu}^{*}-\partial_{\nu} Z_{\mu}^{*}\right)
$$

instead of the fermionic ones. It has been shown [6] that these states with analogous interactions are present among hadron resonances. Such type of new electroweak heavy bosons $Z^{*}$ could also be interesting objects for experimental searches due to their different couplings to the ordinary fermions in comparison with the gauge $Z^{\prime}$ couplings. Alas, this proposal is not popular now and is not present in the experimental programs, yet. With this note we would like to establish the status quo of our alternative proposal for the search for new spin-1 heavy bosons.

In what follows we will concentrate only on resonance production of the excited neutral heavy bosons at the CERN LHC and their search via the very clean dilepton Drell-Yan process. To be more concrete in our predictions we will use a simple phenomenological model of excited bosons with the chiral couplings as in eq. (2).

\section{THE MODEL}

New heavy neutral gauge bosons are predicted in many extensions of the Standard Model (SM). They are associated with additional $U(1)^{\prime}$ gauge symmetries and are generically called $Z^{\prime}$. The gauge interactions of these bosons with matter lead to a specific angular distribution of the outgoing lepton in the dilepton center-of-mass reference frame with respect to the incident parton

$$
\frac{\mathrm{d} \sigma_{Z^{\prime}}}{\mathrm{d} \cos \theta^{*}} \propto 1+\mathrm{ASYM} \cdot \cos \theta^{*}+\cos ^{2} \theta^{*},
$$

which at present is interpreted as a canonical signature for the intermediate bosons with spin 1 . The coefficient ASYM defines the backward-forward asymmetry, depending on $P$-parity of $Z^{\prime}$ couplings to matter.

In addition, another type of spin-1 bosons may exist, which leads to a different signature in the angular distribution. This follows from the presence of different types of relativistic spin- 1 fermion chiral currents $\bar{\psi} \gamma^{\mu}\left(1 \pm \gamma^{5}\right) \psi$ and $\partial_{\nu}\left[\bar{\psi} \sigma^{\mu \nu}\left(1 \pm \gamma^{5}\right) \psi\right]$, which can couple to the corresponding bosons. The hadron physics of the quarkantiquark meson states provides us with an example of such kind of interactions and a variety of spin-1 states.

It was pointed out [6] that three different quantum numbers $J^{P C}$ of existing spin-1 mesons, $1^{--}, 1^{++}$ and $1^{+-}$, cannot be assigned just to two vector $\bar{q} \gamma^{\mu} q$ 
and axial-vector $\bar{q} \gamma^{\mu} \gamma^{5} q$ quark states. So, the additional antisymmetric quark tensor currents $\partial_{\nu}\left(\bar{q} \sigma^{\mu \nu} q\right)$ and $\partial_{\nu}\left(\bar{q} \sigma^{\mu \nu} \gamma^{5} q\right)$ are required, which also describe vector and axial-vector meson states, but with different transformation properties with respect to Lorentz group and with different quantum numbers $1^{--}$and $1^{+-}$, respectively. This example demonstrates that both the pure tensor states, $b_{1}$ mesons, and mixed combinations of vector and tensor states, $\rho$ and $\rho^{\prime}$ mesons, exist.

The mesons coupled to the tensor quark currents are some types of "excited" states as far as the only orbital angular momentum with $L=1$ contributes to the total angular moment, while the total spin of the system is zero. This property manifests itself in their derivative couplings to matter and a different chiral structure of the interactions in comparison with the gauge ones. In contrast with the gauge couplings, where either only lefthanded or right-handed fermions participate in the interactions, the tensor currents mix both left-handed and right-handed fermions. Therefore, like the Higgs particles the corresponding bosons carry a nonzero chiral charge. To our knowledge, such bosons were first introduced by Kemmer [7] and they naturally appear in the extended conformal supergravity theories [8].

There are searches for the excited lepton and quark states, but not for the boson ones. This paper fills this gap considering properties of the neutral heavy chiral bosons which help us to disentangle their production at the hadron colliders from other particles. In order to detect them in the Drell-Yan processes they should couple to the down type of fermions

$$
\mathcal{L}_{\text {excited }}=\frac{g}{2 \sqrt{2} \Lambda}\left(\bar{\ell} \sigma^{\mu \nu} \ell+\bar{d} \sigma^{\mu \nu} d\right)\left(\partial_{\mu} Z_{\nu}^{*}-\partial_{\nu} Z_{\mu}^{*}\right) .
$$

Let us assume for simplicity that $\Lambda$ is equal to the mass of the new $Z^{*}$ boson

$$
M \approx 1 \mathrm{TeV}
$$

and $g$ being the coupling constant of the $S U(2)_{W}$ weak gauge group. For comparison we will consider topologically analogous gauge interactions of the $Z^{\prime}$ boson

$$
\mathcal{L}_{\text {gauge }}=\frac{g}{2}\left(\bar{\ell} \gamma^{\mu} \ell+\bar{d} \gamma^{\mu} d\right) Z_{\mu}^{\prime}
$$

with the same mass $M$. The coupling constants are chosen in such a way that all fermionic decay widths in the Born approximation of the both bosons are identical. It means that their total production cross-sections at the hadron colliders are nearly equal up to next-to-leading order corrections. Their leptonic decay width

$$
\Gamma_{\ell}=\frac{g^{2}}{48 \pi} M \approx 2.8 \mathrm{GeV}
$$

is sufficiently narrow so that they can be identified as resonances at the hadron colliders in the Drell-Yan process.

\section{THE EXPERIMENTAL SIGNATURE}

Up to now, the excess in the Drell-Yan process with high-energy invariant mass of the lepton pairs remains the clearest indication of the heavy boson production at the hadron colliders. So, using only a modest integrated luminosity of $200 \mathrm{pb}^{-1}$ collected during RUN II, the D0 Collaboration puts tight restrictions on the $Z^{\prime}$ masses for the different models from the dielectron events [10]: $M_{Z_{S M}^{\prime}}<780 \mathrm{GeV}, M_{Z_{\eta}^{\prime}}<680 \mathrm{GeV}, M_{Z_{\psi}^{\prime}}<650 \mathrm{GeV}$, $M_{Z_{x}^{\prime}}<640 \mathrm{GeV}$ and $M_{Z_{I}^{\prime}}<575 \mathrm{GeV}$. Comparable statistics in the dimuon channel leads approximately to the same constraint $M_{Z_{S M}^{\prime}}<680 \mathrm{GeV}$ [11]. The CDF constraints from the dielectron channel are based on more data, $1.3 \mathrm{fb}^{-1}$, which leads to tighter restrictions [12]: $M_{Z_{S M}^{\prime}}<923 \mathrm{GeV}, M_{Z_{\eta}^{\prime}}<891 \mathrm{GeV}, M_{Z_{\psi}^{\prime}}<822 \mathrm{GeV}$, $M_{Z_{\chi}^{\prime}}<822 \mathrm{GeV}$ and $M_{Z_{I}^{\prime}}<729 \mathrm{GeV}$. Therefore, our mass choice (5) for the new bosons does not contradict the Tevatron constraints. At the same time such bosons could be observed as resonance peaks on the $Z$ boson tail in the invariant dilepton mass distribution at the LHC (Fig. 1 [14]) already in the first days of the physical runs.

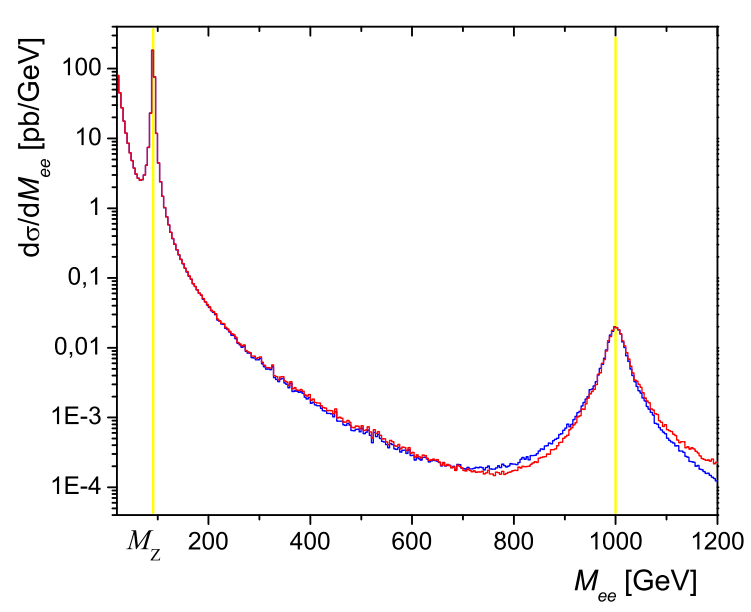

FIG. 1: The invariant dilepton mass distributions for the gauge $Z^{\prime}$ boson (blue) and the excited chiral $Z^{*}$ boson (red) with the Drell-Yan SM background at the CERN LHC.

The peaks in the invariant mass distributions originate from the Breit-Wigner propagator form, which is the same both for the gauge and chiral bosons in the Born approximation. However, the common wisdom, that a peak in the invariant mass distribution of the two final particles must correspond to the Jacobian peaks in their transverse momentum distributions $p_{T}$, is not valid for the chiral bosons due to the following fact. The main feature of the interactions (44) consists in different angu- 
lar distribution of final fermions []

$$
\frac{\mathrm{d} \sigma_{Z^{*}}}{\mathrm{~d} \cos \theta^{*}} \propto \cos ^{2} \theta^{*}
$$

in comparison with the distribution (3) for the gauge interactions. It leads to a stepwise lepton transverse momentum distribution, rather than to the Jacobian peak at the kinematical endpoint $M / 2$ for the gauge bosons (Fig. 2). Therefore, already the lepton transverse mo-

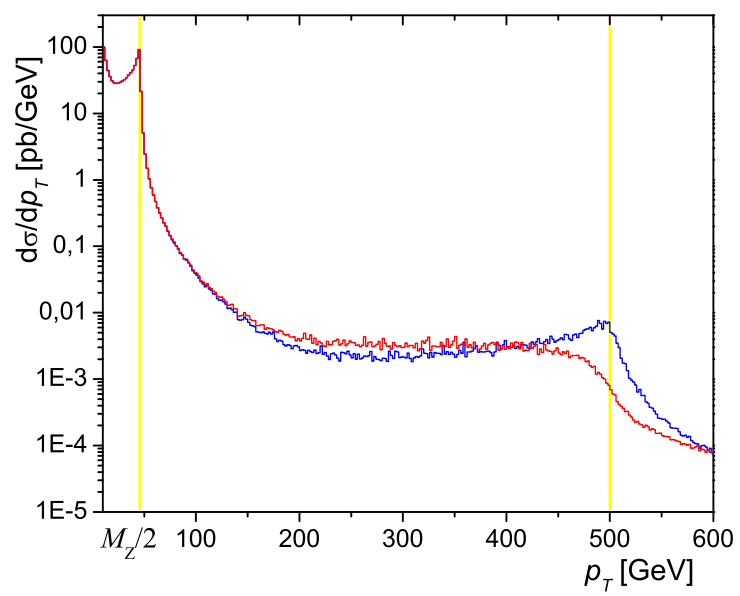

FIG. 2: The differential cross-sections for the gauge $Z^{\prime}$ boson (blue) and the excited chiral $Z^{*}$ boson (red) with the Drell-Yan SM background as functions of the lepton transverse momentum at the CERN LHC.

mentum distribution demonstrates a difference between the gauge and chiral bosons. In order to make more substantial conclusions, let us investigate other distributions selecting only "on-peak" events with the invariant dilepton masses in the range $800 \mathrm{GeV}<M_{\ell \ell}<1200 \mathrm{GeV}$.

According to the eq. (8), there exists a characteristic plane, perpendicular to the beam axis in the parton rest frame, where the emission of the final-state pairs is forbidden. The nonzero probability in the perpendicular direction in the laboratory frame is due to the longitudinal boosts of colliding partons. So, at the Fermilab Tevatron the production of such heavy bosons occurs almost at the threshold with approximately zero longitudinal momenta. Hence, the lepton pseudorapidity distribution for the chiral bosons has a minimum at $\eta_{\ell}=0$ (Fig. 3). On the other hand the CERN LHC is sufficiently powerful to produce heavy bosons with a mass $M=1 \mathrm{TeV}$ with high longitudinal boosts. Therefore, the pseudorapidity distributions for the gauge and chiral bosons at the LHC look similar (Fig. 4).

Crucial confirmation of the existence of the new interactions (4) should come from the analysis of the angular distribution of the final leptons with respect to the boost direction of the heavy boson in the rest frame of the latter

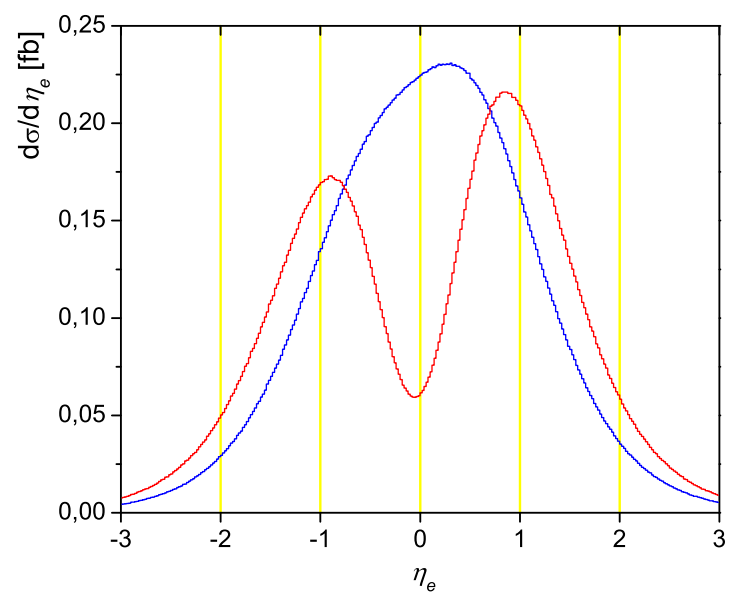

FIG. 3: The differential cross-sections for the gauge $Z^{\prime}$ boson (blue) and the excited chiral $Z^{*}$ boson (red) decaying to a lepton pair with the invariant mass $800 \mathrm{GeV}<M_{\ell \ell}<1200 \mathrm{GeV}$ as functions of the lepton pseudorapidity at the Fermilab Tevatron.

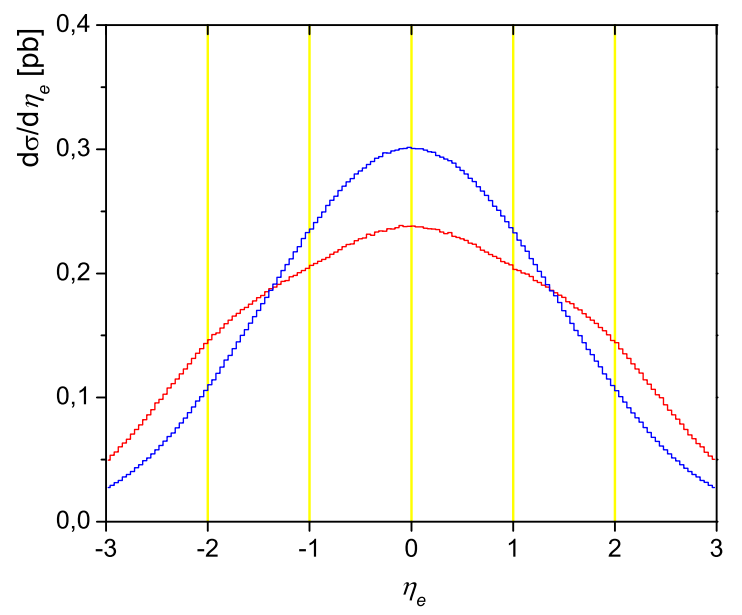

FIG. 4: The differential cross-sections for the gauge $Z^{\prime}$ boson (blue) and the excited chiral $Z^{*}$ boson (red) decaying to a lepton pair with the invariant mass $800 \mathrm{GeV}<M_{\ell \ell}<1200 \mathrm{GeV}$ as functions of the lepton pseudorapidity at the CERN LHC.

(the Collins-Soper frame). In the Fig. 5 we compare the differential cross-sections for the gauge $Z^{\prime}$ boson and the excited chiral $Z^{*}$ boson decaying to the lepton pairs with the invariant mass $800 \mathrm{GeV}<M_{\ell \ell}<1200 \mathrm{GeV}$ as functions of $\cos \theta_{\mathrm{CS}}^{*}$. Instead of a smoother angular distribution for the gauge interactions, a peculiar "swallowtail" shape of the chiral boson distribution occurs with a dip at $\cos \theta_{\mathrm{CS}}^{*}=0$. It will indicate the presence of the new interactions. Neither scalars nor other particles possess 


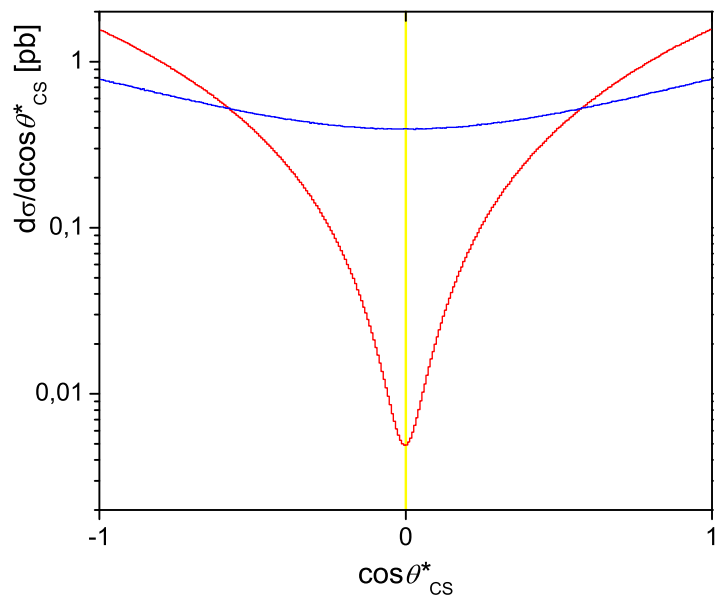

FIG. 5: The differential cross-sections for the gauge $Z^{\prime}$ boson (blue) and the excited chiral $Z^{*}$ boson (red) decaying to a lepton pair with the invariant mass $800 \mathrm{GeV}<M_{\ell \ell}<1200 \mathrm{GeV}$ as functions of $\cos \theta_{\mathrm{CS}}^{*}$ at the CERN LHC. such a type of angular behavior.

\section{CONCLUSIONS}

In this paper we have considered the experimental signatures of the excited chiral heavy bosons $Z^{*}$ and compared them with the gauge $Z^{\prime}$ bosons. It has been stressed that the chiral bosons have a new angular distribution, yet unknown for experimentalists. It leads to an absence of the Jacobian peak in the transverse momentum distribution and to a profound dip in the angular distribution at the rest frame of the heavy chiral boson. These features could help to discriminate the chiral boson production from a resonance production of other particles at the hadron colliders.

\section{Acknowledgements}

We are grateful to V. G. Kadyshevsky, M. D. Mateev, N. A. Russakovich, I. R. Boyko and R. V. Tsenov for support and fruitful cooperation.
[1] ATLAS Collaboration, Detector and Physics Performance, Technical Design Report, CERN/LHCC/99-15, CERN (1999).

[2] G. L. Bayatian et al. (CMS Collaboration), J. Phys. G 34, 995 (2007).

[3] R. Barate et al. (ALEPH Collaboration), Eur. Phys. J. C 4, 571 (1998); G. Abbiendi et al. (OPAL Collaboration), Phys. Lett. B 544, 57 (2002); P. Achard et al. (L3 Collaboration), Phys. Lett. B 568, 23 (2003); J. Abdallah et al. (DELPHI Collaboration), Eur. Phys. J. C 46, 277 (2006).

[4] C. Adloff et al. (H1 Collaboration), Phys. Lett. B 548, 35 (2002); S. Chekanov et al. (ZEUS Collaboration), Phys. Lett. B 549, 32 (2002).

[5] D. Acosta et al. (CDF Collaboration), Phys. Rev. Lett. 94, 101802 (2005); V. M. Abazov et al. (D0 Collaboration) arXiv:0801.0877 [hep-ex].
[6] M. V. Chizhov, arXiv:hep-ph/9610220 M. V. Chizhov, JETP Lett. 80, 73 (2004) arXiv:hep-ph/0307100].

[7] N. Kemmer, Proc. Roy. Soc. A 166, 127 (1938).

[8] Supergravities in Diverse Dimensions, eds. A. Salam and E. Sezgin (North-Holland and World Scientific, 1989).

[9] M. V. Chizhov, arXiv:hep-ph/0008187.

[10] D0 Collaboration, D0 note 4375-CONF (2004).

[11] D0 Collaboration, D0 note 4577-CONF (2004).

[12] T. Aaltonen et al. (CDF Collaboration), Phys. Rev. Lett. 99, 171802 (2007); arXiv:0707.2524 [hep-ex].

[13] A. Pukhov et al., Preprint INP MSU 98-41/542, arXiv: hep-ph/9908288 A. Pukhov, arXiv:hep-ph/0412191

[14] Here and in the following the CalcHEP 13 package will be used for the numeric calculations of various distributions with a CTEQ6M choice for the proton parton distribution set. 